\title{
A Design of Autonomous Goods Transport System Based on Quadcopter
}

\author{
Fengru $\mathrm{Li}^{1, \mathrm{a}}$, Ying Zhong ${ }^{2, \mathrm{~b}}$, Zhixiong $\mathrm{LI}^{3, \mathrm{c}}$, Jinbao Zhu ${ }^{4, \mathrm{~d}}$, Chenghui Qian ${ }^{5, \mathrm{f}}$ \\ ${ }^{1}$ College of Instrument \& Electrical Engineering, Jilin University, Changchun 130000, China \\ ${ }^{2}$ College of Instrument \& Electrical Engineering, Jilin University, Changchun 130000, China \\ ${ }^{3}$ College of Instrument \& Electrical Engineering, Jilin University, Changchun 130000, China \\ ${ }^{4}$ College of Instrument \& Electrical Engineering, Jilin University, Changchun 130000, China \\ ${ }^{5}$ College of Instrument \& Electrical Engineering, Jilin University, Changchun 130000, China \\ a shihun@126.com, ${ }^{b} 284189995 @ q q . c o m,{ }^{c} 448066326 @ q q . c o m,{ }^{d} 1020243577 @ q q . c o m,{ }^{f}$ qianch \\ @jlu.edu.cn
}

Keywords: quadcopter; PID; GNSS; STM32

\begin{abstract}
A quadcopter controlled by PID which uses STM32 as its main control unit and can achieve autonomous goods transporting and positioning with GNSS (Global Navigation Satellite System) has been designed. To control its attitude, a dynamic model has been describe with the collected data of attitude of quadcopter, combined with Euler angles. The acquisition information is transmitted wirelessly to the host computer, according to the algorithm, adjust the control parameters to shorten response time, and make the system highly stable. Test results show that the control algorithm can make the quadcopter stable flight. The accuracy of goods delivery is 2 meters.
\end{abstract}

\section{Introduction}

In recent years, extensive use of unmanned aircraft has become a hot topic in many countries, the United States, Japan, France and other countries have such a concept research project [1]. Because market-oriented civilian aircrafts have many disadvantage such as expensive, single function, hard to manipulate and active control, internal research and development are relatively slow [2]. In view of this, a quad-rotor with autonomous goods transporting by using GNSS positioning has been designed. To make quadcopter fly stably, a dynamic model has been described, and the PID algorithm has been used to control the quadcopter. In addition to stably flying, the quadcopter can get positioning information of GNSS modules to find the target position, and land after reaching the target position.

\section{Principle and Algorithm}

\section{Dynamics Modeling.}

The structure of quadcopter is " $\mathrm{X}$ " type , compared with the "+" type structure, it has great ability of airborne and can adjust the space attitude flexibly, realizing the quadcopter's take-off and landing, hovering, pitch, roll and yaw motion, etc.

1) Establishment of the equivalent model

The quadcopter adjust the attitude by changing the rotor speed to change the counterbalance of lift and gravity .Quadcopter is one who has a six degrees of freedom of movement and four controllable input less drive systems. The dynamic tensile force $\mathrm{f}$ is proportional to the square of the angular velocity, with a positive direction constantly. As shown in figure 1: 


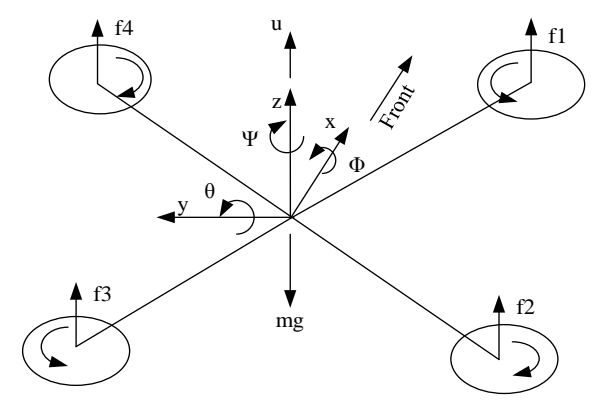

Figure 1. quadcopter's structure diagram and stress analysis

$f_{i}=\frac{1}{2} \rho \cdot C_{T} \cdot A_{i} \cdot\left(\omega \cdot R_{i}\right)^{2}$

In formula 1, $\rho$ is air density, $C_{T}$ is drag coefficient, $A_{i}$ is the area for the $\mathrm{i}$-th propeller, $\omega_{i}$ is the motor's rotation speed belongs to the $\mathrm{i}$-th propeller, $\mathrm{R}_{i}$ is the radius length of the $\mathrm{i}$-th rotor blade .Due to the motor, rotor blade size and material are same, the $\rho, C_{T} 、 \mathrm{R}_{i}$ can approximate to constant parameters. Tension produced by four propeller is proportional to the square of angular velocity, so formula 1 formula can be simplified into 2 .

$f_{i}=k \cdot \omega^{2}$

From force analysis to model we can carry out that the combined tension $u$ is the sum of each individual rotor tension. The pitch torque $\tau_{\theta}$ is the sum of motor $M_{2}$ and motor $M_{3}$ tension subtract the sum of motor $\mathrm{M}_{1}$ and motor $\mathrm{M}_{4}$ tension. $\tau_{\theta}$ is shown as the formula 3.

$\tau_{\theta}=\left[\left(f_{1}+f_{4}\right)-\left(f_{2}+f_{3}\right)\right] \cdot l$

In formula 3,1 is the perpendicular distance from the center of the adjacent two motor's shaft to the quadcopter's center of gravity. The roll torque $\tau_{\phi}$ is the sum of motor $\mathrm{M}_{2}$ and motor $M_{1}$ tension subtract the sum of motor $M_{3}$ and motor $M_{4}$ tension. $\tau_{\phi}$ is shown as the formula 4.

$\tau_{\phi}=\left[\left(f_{1}+f_{2}\right)-\left(f_{3}+f_{4}\right)\right] \cdot l$

The yaw torque $\tau_{\psi}$ is the sum of each reacting torque $\tau_{\psi} \mathrm{M}_{i}$ produced by the propeller. $\tau_{\psi}$ is shown as the formula 5 .

$\tau_{\psi}=\tau_{M_{1}}+\tau_{M_{2}}+\tau_{M_{3}}+\tau_{M_{4}}$

When set the resistance torque of motor to $\tau_{\text {drag }}$ and rotation torque is defined as formula 6

$I_{\text {rot }} \cdot \omega_{i}=\tau_{M i}-\tau_{\text {dragi }}$

$I_{\text {rot }}$ is the moment of inertia for motor rotor goes around its own axis. $\omega_{i}$ is the angular velocity of the motor rotor. $\tau_{M i}-\tau_{\text {dragi }}$ is the combining torque. According to the aerodynamics the definition of drag torque is shown as formula 7.

$\tau_{\text {dragi }}=\frac{1}{2} \rho \cdot A_{i} \cdot v_{i}^{2}$

$v_{i}$ is the $\mathrm{i}$-th speed of propeller.Formula 7 can be simplified to formula 8 .

$\tau_{\text {dragi }}=K_{\text {drag }} \cdot \omega_{i}^{2}$

$K_{\text {drag }}>0$ is a constant depends on the air density.For quasi stationary quadcopter flight movement $\omega$ is a constant. Finally we can get formula 9.

$\tau_{M i}=\tau_{\text {dragi }}$

Brief introduction of flight attitude.

Vertical flight: Increasing the four motor output power at the same time to strengthen the tensile force u. Quadcopter will fly upwards when $\mathrm{u}>\mathrm{mg}$. As shown in figure 2. 


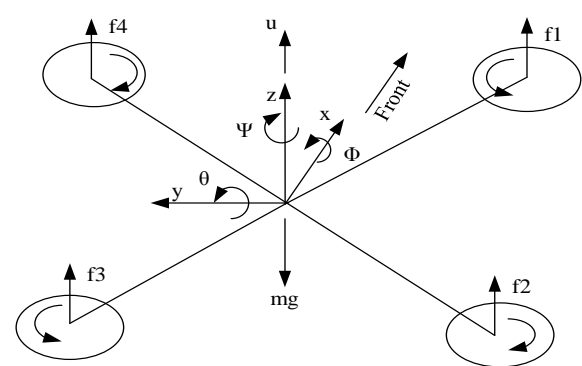

Figure 2. Quadcopter's vertical flight

- Forward and backward flight: Making M2 and M3 motor speed increase so that the force $\mathrm{f} 2+\mathrm{f} 3$ will increase. At the same time, making M1 and M4 motor speed decrease so that the force $\mathrm{f} 1+\mathrm{f} 4$ Will decrease .When $(\mathrm{f} 2+\mathrm{f} 3)-(\mathrm{f} 1+\mathrm{f} 4)>0$ the quadcopter can get the force to fly forward.

- Tumbling flight: Making M1 and M2 motor speed increase so that the force $\mathrm{f} 1+\mathrm{f} 2$ will increase. At the same time, making M3 and M4 motor speed decrease so that the force $\mathrm{f} 1+\mathrm{f} 4$ will decrease .When $(\mathrm{f} 1+\mathrm{f} 2)-(\mathrm{f} 3+\mathrm{f} 4)>0$ the quadcopter can get the force to make tumbling flight.

- Yawed flight: Control the M1 and M3, and make the rising tension $\mathrm{f} 1$ and $\mathrm{f} 3$ increase. At the same time control the M1 and M3, make the rising tension f2 and f4 decrease. Making sure that the total pulling force $\mathrm{u}$ equals to $\mathrm{mg}(\mathrm{u}=\mathrm{mg})$ and then the quadcopter can make yawed flight.

Quaternion.

Quaternion is the main and most fundamental in the process of control algorithm. Due to that the precision of attitude algorithm is the premise to realize stable flight, while many small quadcopter use micro-electro-mechanical systems (MEMS) devices, the problem such as low accuracy, easily diverging, restricting the navigation features of the stability of the aircraft. For this purpose, quaternion compensation algorithm is used. This algorithm is simple and effective, less demanding of accuracy of device. The attitude can maintain stable for a long time after dealing with this algorithm. Here is a brief introduction to the method of using quaternion to obtain the Euler angles.

Nitialization of quaternion:

$\mathrm{q}=\left[\begin{array}{llll}q_{0} & q_{1} & q_{2} & q_{3}\end{array}\right]^{T}=\left[\begin{array}{llll}1 & 0 & 0 & 0\end{array}\right]^{T}$

The acceleration and angular velocity was obtained from the gyroscope: three axis acceleration $\mathrm{a}_{x}, \mathrm{a}_{y}, \mathrm{a}_{z}$ three axis angular velocity $\mathrm{g}_{x}, \mathrm{~g}_{y}, \mathrm{~g}_{z}$.

Change $\mathrm{a}_{x}, \mathrm{a}_{y}, \mathrm{a}_{z}$ to three dimensional unit vector(standardization), the result is:

$a_{x}=\frac{a_{x}}{\sqrt{a_{x}^{2}+a_{y}^{2}+a_{z}^{2}}}$

$\mathrm{a}_{y}$ and $\mathrm{a}_{z}$ are same as above.

Obtain three axis of gravity from quaternion :

$v_{x}=2\left(q_{1} q_{3}-q_{0} q_{2}\right)$

$v_{y}=2\left(q_{0} q_{1}-q_{2} q_{3}\right)$

$v_{z}=q_{0}^{2}-q_{1}^{2}-q_{2}^{2}+q_{3}^{2}$

The error is the difference of exterior product $\vec{e}=\vec{a} \times \vec{y}$, used as correction of gyroscope.

$e_{x}=a_{y} \cdot v_{z}-a_{z} \cdot v_{y}$

$e_{y}=a_{z} \cdot v_{x}-a_{x} \cdot v_{z}$

$e_{z}=a_{x} \cdot v_{y}-a_{y} \cdot v_{x}$

Use the error as correction of gyroscope:

$e_{\text {xint }}=k_{i} \int_{0}^{t} e_{x} d t+k_{i} \int_{t-1}^{t} e_{x} d t$

$e_{y i n t}$ and $e_{\text {zint }}$ are same as above.

$g_{x i n t}=g_{x}+k_{p} \cdot e_{x}+k_{i} \int_{0}^{t} e_{x} d t$

$g_{y i n t}$ and $g_{z i n t}$ are same as above.

Use the correction of gyroscope to update quaternion :

$q_{0}=q_{0}+\frac{T}{2}\left(-q_{1} g_{x i n t}-q_{2} g_{y i n t}-q_{3} g_{\text {zint }}\right)$ 
$q_{1}=q_{1}+\frac{T}{2}\left(q_{0} g_{x i n t}+q_{2} g_{y i n t}-q_{3} g_{z i n t}\right)$

$q_{2}=q_{2}+\frac{T}{2}\left(q_{0} g_{x i n t}-q_{1} g_{y i n t}+q_{3} g_{\text {zint }}\right)$

$q_{3}=q_{3}+\frac{T}{2}\left(q_{0} g_{x i n t}-q_{2} g_{y i n t}-q_{3} g_{\text {zint }}\right)$

Make the new quaternion normalization:

$q_{0}=\frac{q_{0}}{\sqrt{q_{0}^{2}+q_{1}^{2}+q_{2}^{2}+q_{3}^{2}}}$

$q_{1}, q_{2}$ and $q_{3}$ are same as above.

Above-mentioned complete a quaternion update, use it as the next initial quaternion. Chang all quaternions to three Euler angles, complete the preliminary operation of attitude.

$\begin{aligned} \varphi & =\tan ^{-1}\left(\frac{2\left(q_{2} q_{3}+q_{0} q_{1}\right)}{q_{0}^{2}-q_{1}^{2}-q_{2}^{2}+q_{3}^{2}}\right) \\ \theta & =\sin ^{-1}\left(2\left(q_{1} q_{3}-q_{0} q_{2}\right)\right) \\ \psi & =\tan ^{-1}\left(\frac{2\left(q_{1} q_{2}+q_{0} q_{3}\right)}{q_{0}^{2}+q_{1}^{2}+q_{2}^{2}+q_{3}^{2}}\right)\end{aligned}$

The PID control algorithm.

The quadcopter use PID control algorithm. The quadcopter is nonlinear system but it can approximate to linear system. PID controller is a common controller in industrial control .It consist of proportion element Integral element I, and differentiation element D. Time domain differential equation for the PID controller:

$\mathrm{u}(\mathrm{t})=K_{p}\left[e(t)+\frac{1}{T_{i}} \int e(t) d t+T_{d} \frac{d e(t)}{d t}\right]$

$\mathrm{K}_{p}$ is proportionality coefficient, $\mathrm{T}_{i}$ is integral time constant, $\mathrm{T}_{d}$ is differentiating time constant. $\mathrm{U}(\mathrm{k})$ is the discretization equation of PID controller :

$\mathrm{u}(\mathrm{t})=K_{p} e(k)+K_{i} \sum_{i=0}^{k} e(i)+K_{d}[e(k)-e(k-1)]$

$K_{i}=\frac{K_{p} T}{T_{i}}$ is integral coefficient, $K_{d}=\frac{K_{p} T_{d}}{T}$ is differential coefficient. $\mathrm{T}$ is sampling time.The principle of PID control block diagram as shown in figure 3 :

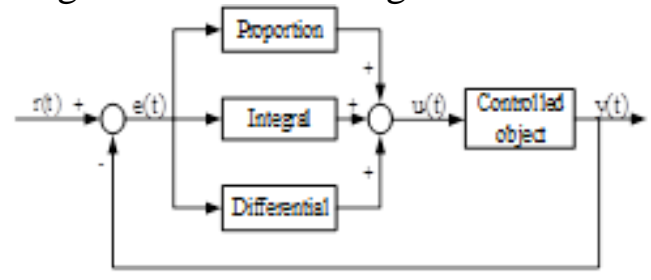

Figure 3. A typical PID control system structure diagram

\section{The Kalman Filter algorithm.}

1)Establishing model for quadcopter attitude

Kalman filter is an algorithm that uses linear system's state equation and the input and output data to make optimum estimation for system state. Due to the observation data include noise and interference of the system, the operation of making optimum estimation is seen as a filtering process.

State equation:

$x_{k+1}=A x_{k}+B u_{k}+q_{k}$

Output equation:

$y_{k}=C x_{k}+r_{k}$

$\mathrm{k}$ is time factor,A,B and C is matrix, $\mathrm{x}$ is state variable, $\mathrm{u}$ is known inputs of the system,y is output signal,q is process noise, $\mathrm{r}$ is measurement noise[6].

2) Kalman filtering algorithm implementation steps

Since the sampling and operation time in the process of flight are very short, we can consider the changes of angle as linear. Processing signal through Kalman filtering algorithm can reduce noise. 
The process of filtering the gyroscope data is as follows:

Get the current gyroscope data $\mathrm{u}$ and angle y gained through acceleration sensor;

According to the system model, we can estimate the current state of the system from the last moment:

$\mathrm{X}(\mathrm{k} \mid \mathrm{k}-1)=\mathrm{A} \cdot \mathrm{X}(\mathrm{k}-1 \mid \mathrm{k}-1)+\mathrm{B} \cdot \mathrm{U}(\mathrm{k})$

Update Covariance:

$\mathrm{P}(k \mid k-1)=A \cdot P(k-1 \mid k-1) A^{T}+\mathrm{Q}$

$\mathrm{P}(\mathrm{k} \mid \mathrm{k}-1)$ is the corresponding covariance of $\mathrm{A}(\mathrm{k} \mid \mathrm{k}-1), \mathrm{P}(\mathrm{k}-1 \mid \mathrm{k}-1)$ is the covariance of $\mathrm{k}-1$ optimal system state in the k-1 moment, AT is the transpose matrix of A, and Q is the covariance of system processing.

Calculate the Gain of Kalman:

$K_{g}(k)=\frac{P(k \mid k-1) H^{T}}{H \cdot P(k \mid k-1) H^{T}+R}$

Get the optimal estimation of the current state:

$\mathrm{X}(k \mid k)=X(k \mid k-1)+K_{g}(k)(Z(k)-H \cdot X(k \mid k-1))$

Update the covariance of system:

$\mathrm{P}(k \mid k)=\left(I-K_{g}(k) H\right) P(k \mid k-1)$

\section{Hardware Design and Simulation}

\section{The structure of main parts.}

The fuselage uses F450 chassis, and the dynamic system uses electronic governor combined with brushless DC motors. The electronic governor (hereinafter referred to as ESC) inverts DC current into three-phase alternating current by the way of no-inductance commutation and provides which to the brushless DC motors. The UBLOX series GNSS position module NEO-M8N is used for that it has a high precision of positioning. To the part of fixed height, the ultrasonic module is chosen which can adjust its measurement range by the value of a resistance and the maximum measurement range can achieve $450 \mathrm{~cm}$.

\section{The use of main chips.}

The Quadcopter has a higher acquirement to controller than other control system. The operation process needs faster acquisition speed, shorter response time and lower power. Therefore STM32F103ZET6 is used as the MCU, which is a processor of Cortex-M3 kernel of ST.LIB and its kernel is based on ARMv7 series structure. The MPU6050 is used to measure the tri-axis acceleration and angular velocity. It has characteristics of high precision, the high speed of acquisition and calculation in practical use, and meet the need of the acquirement that the MCU need a high sensitivity response of flight attitude. The yaw angle and the orientation of quadcopter are needed to determined and measure. So the HMC5883L, an electronic compass with an integrated circuit in Honeywell patent including amplifier and automatic degaussing driver. The error of the compass can be controlled in $1^{\circ} \sim 2^{\circ}$ and it has an interface of simple I2C series bus. However, the compass is easy to be interfered by magnetic field so the quadcopter need to be rotated around the each axis to adjust the magnetometer.

\section{The hardware main framework.}

To divide the module according to the different functions, the system can be divided into two part, the main control part and the power supply part. 


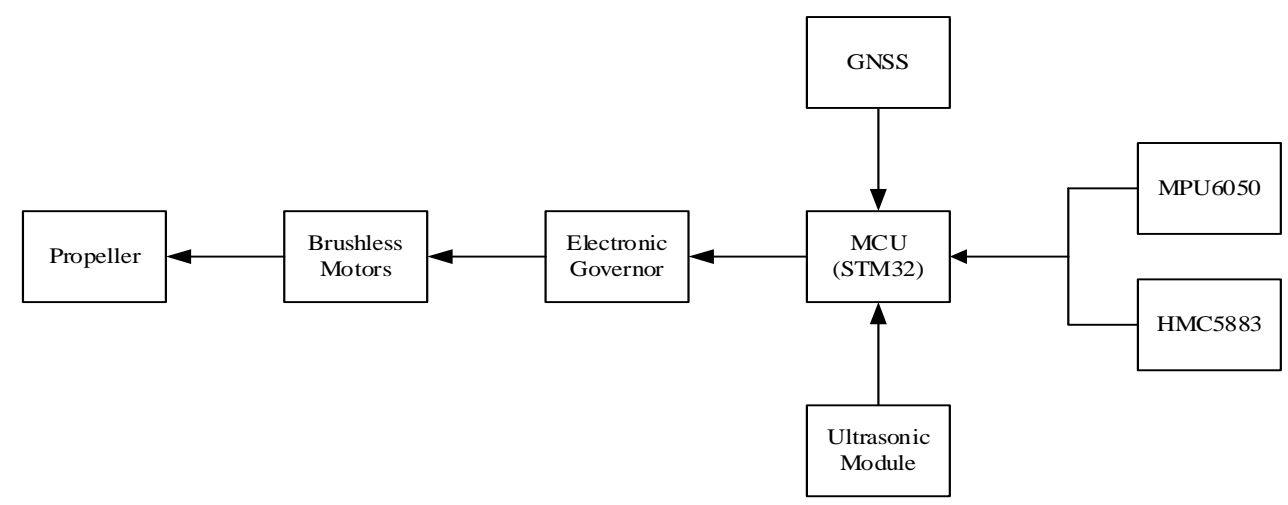

Figure 5. Master control parts diagram

\section{The design of software}

The attitude-control program.

The software of the control system is written in language C. The main function is not only to control the flight direction according to the data which the GNSS received, but also to close loop control the flight according to the data of the attitude of flight collected. The cascade PID that an inner PID and an outer PID is used in the control program. The PID output of outer loop that the expected value, is given to the inner loop to calculate of PD. After the attitude of calculating and filtering, there is deviation between the angle of output and the initial angle. PID controller is needed to decrease the deviation by the four brushless DC motors. To take the pitch angle as an example:

To suppose the quiescent value initially is pitch.offset, the measurement value measured by sensor is PITCH. So the deviation is $\mathrm{e}(\mathrm{k})=\mathrm{PITCH}-$ pitch.offset. When the deviation is integrated pit.increment $+=\mathrm{e}(\mathrm{k})$

However, it is easy to achieve the saturation of the deviation. So an integrated limiting is used. $\mid$ pit.increment $\mid<=$ pit.increment.max

The deviation is $\mathrm{e}(\mathrm{k})-\mathrm{e}(\mathrm{k}-1)$. So the output of PID controller is Pidout=kp*e(k)+ki*pit.increment+kd*[e(k)-e(k-1)].

Eventually, PID adjusting pitch, roll and yaw and then output 4-channel PWM wave combined with throttle, which control the speed of 4 motors. Due to the vibration effects of 4 motors and fuselage, three-axis angular velocity $\omega$ data contains a lot of noise. While the gyroscope is less affected by noise, accelerometer data must be for Kalman filtering to remove noise and then to have data fusion with gyroscope data in order to eliminate the accumulated error of gyroscope.

GNSS.

GNSS (Global Navigation Satellite System), is using GPS, GLONASS, Galileo, QZSS, Beidou satellite positioning systems for integrated positioning. It has many advantages such as more online satellites and higher accuracy. The data from GNSS is read in MCU by serial port, and GNSS gets 7 mode data by means of satellite position. So "GNRMC" is the recommended format. After analyzing the return data, MCU takes current coordinates and coordinates of destination for comparison and calculation. Combined with magnetometers that have the orientation of the flight to change the yaw angle, and makes the handpiece have always been the destination.

\section{Ultrasonic fixed height system.}

PID regulating mode is used for ultrasonic part. PID part in attitude control section has detailed instructions, and not to repeat here. For brushless motors, three-phase voltage is proportional to its speed of motor.

$\omega=\mathrm{KV} * \mathrm{U}$ 
Because the purpose of this design is to realize the delivery, the flight time is long, the supply voltage will be reduced to a certain extent during the flight. However electronic speed control output voltage is proportional to the voltage of the power supply. Being measured at different voltages the electronic speed control output voltage as shown in Figure 6 and Figure 7. Therefore, a voltage control is needed to be added in fixed high part. Its output of PWM duty cycle is inversely proportional to the voltage at the moment. After joining this voltage for PWM control, during a long flights, quadcopter has a downward acceleration due to the voltage drops. The error for ultrasonic fixing height is within $10 \mathrm{~cm}$.

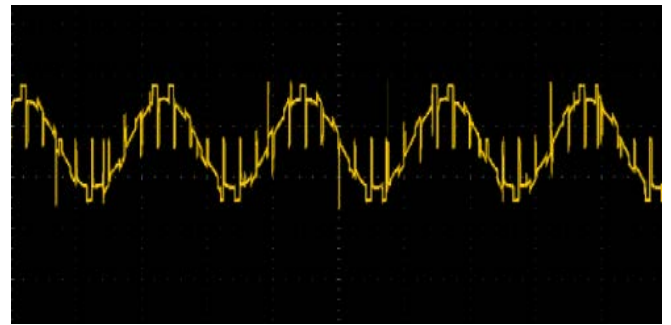

Figure 6.11.5V electronic speed controller output wave form

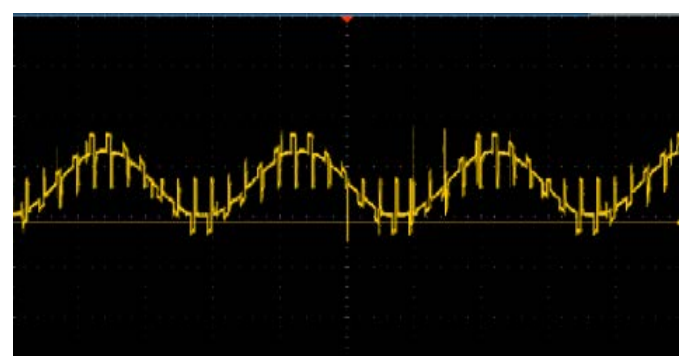

Figure 7.10V electronic speed controller output wave form

\section{Actual test and analysis}

\section{Kalman filtering algorithm.}

Established model of aircraft flight time, and the two parameters of this model to calculate the Kalman filter, and two parameter of Kalman filter can be calculated according to the model. Process noise Q, if Q increases, it will have faster dynamic response, and convergence stability more deteriorate; Measurement noise $\mathrm{R}$, if $\mathrm{Q}$ increases, it will have slower dynamic response, and convergence stability better.

Kalman filter effect diagram as shown in Figure 8, the purple line is the acceleration before the filter, the blue-green line filtered acceleration waveforms.

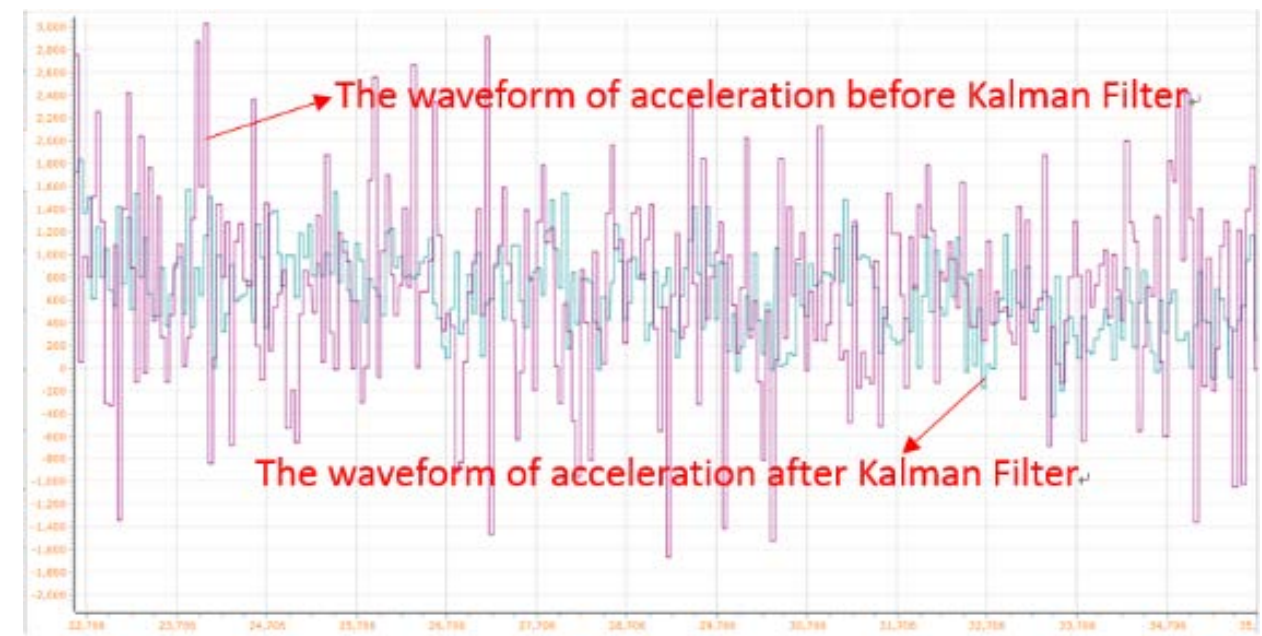

Figure 8. The effects of Kalman filter

The images show that, Kalman filter can effectively filter out motor and gyro noise caused by air vibrations. 


\section{Parameter adjustment of flight attitude algorithm.}

In part of the attitude adjustment, MCU sends attitude data and a variety of sensor data to the PC, and through the serial port to the PC software, and then turns the PID value of quadcopter.

Interference with quadcopter can quickly go back to the equilibrium position, aircraft attitude shocks curve as shown in Figure 9.

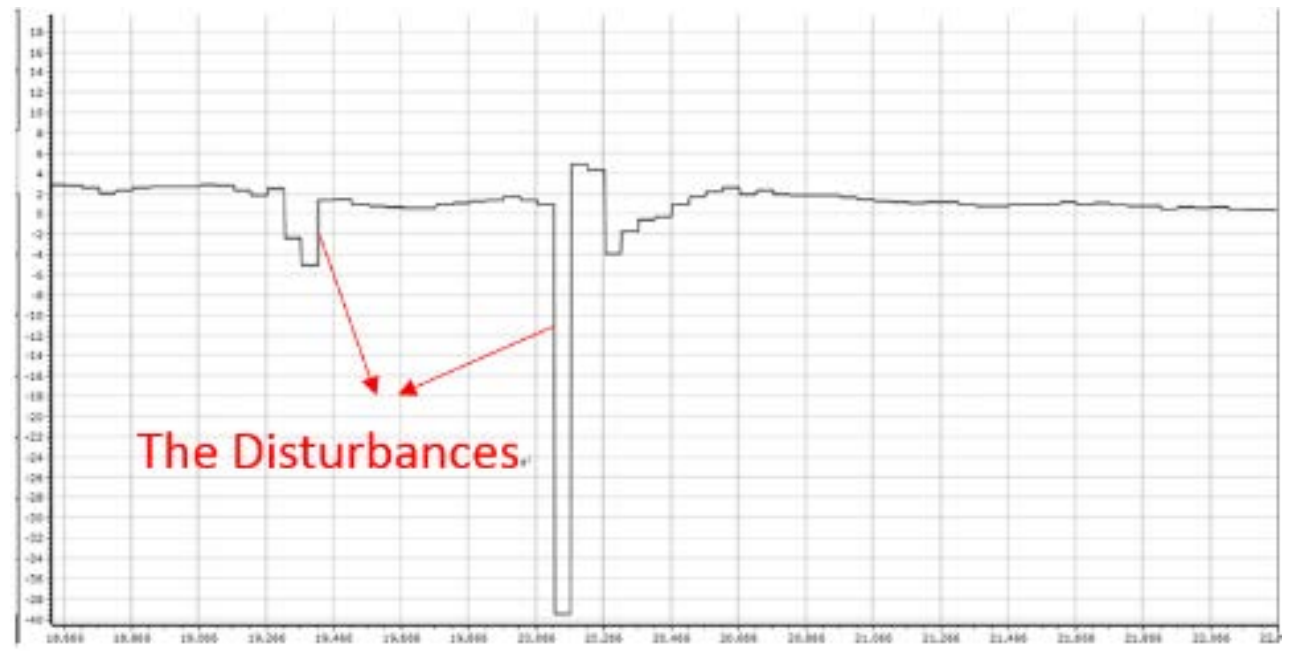

Figure 9 aircraft attitude shock curve

Properly adjusted PID parameters for quadcopter can hover stably attitude. Visible when there is interference, the aircraft can be quickly adjusted to balance stance with less vibrations and better system convergence.

\section{Concluding remarks}

Properly adjusted PID parameters for quadcopter, could not only achieve quadcopter attitude control for smooth flight, but also independent delivery of the aircraft by GNSS and magnetometer data. The design reduces the operation of professionals during the operation, and reduces costs too.

Autonomous quadcopter delivery system, aiming commercial UAV at the market having many shortcomings such as requiring professionals to operate and difficult to control, uses economical and reliable hardware solutions and efficient, energy-saving, stable software solution to make which has the advantages of low cost and high performance, and provides a theoretical basis to some small unmanned aircraft systems in the market.

\section{References}

[1]Hong Sen-tao, Jin Zhi-hui, Li Zhi-qiang. Quadrotor modeling and analysis of posture stability [J]. Electro-optical system, 2008, (2): 34-37.

[2]Nie Bowen. Research status and key technology of microminiature quadrotor[J]. Electronics Optics and Control, 2007, 14 (6):113-117.

[3]SUKTHANKAR R.PCA-SIFT: A more distinctive representation for local image descriptions [C] Proceedings Conference Computer Vision and Pattern Recognition, 2004: 511-517 .

[4]Ma Min, Haichao. Quadrotor posture calculating based on quaternion compensation[M]. Manufa cturing Automation, 2013, 12 (23).

[5]Pang Qingpei, Li Jiawen, Huang Wenhao. Designment of quadrotor and imitation research of trim control [A], electro-optics and control, 2012 (5).

[6]Zhou Xiongrui, Chen Wutong, Chen Mingchun, etc. Kalman Predictor and Multitarget Tracking Algorithm [J]. China Institute of Technology journal, 2003, 5 (26): 22-36. 
[7]Sun Jianzhong, Bai Fengxian. The special motor control[M]. Beijing: China Waterpower Press, 2 005: 37-40.

[8]Li Ning.Development and application of STM32 based on MDK[M], Beijing: Beihang University Press, 2008: 1-4 